\title{
Study of network architecture for Unmanned Aerial Vehicle
}

\author{
Hai-yan Zhao ${ }^{1, ~ a}$, Wen-jing Yang ${ }^{1, b}$, Feng-chen Qian ${ }^{1, c}$, Xiao-shuang Wang ${ }^{1, b}$, Ran Li $i^{1, c}$ \\ ${ }^{1}$ College of Information and Communications, National University of Defense Technology, Xi'an, \\ Shaanxi, 710106, China \\ a51375908@qq.com, b813086903@qq.com, '23414388@qq.com
}

\begin{abstract}
With the rapid development of UAV (unmanned aerial vehicle) technology in the past decade, UAS tend to play an increasingly important role in information acquisition and unmanned operations. It becomes difficult for a single drone to meet the needs of complex tasks, and the application mode of drone formation coordination has gained great attention. First of all, this paper analyzes the structure of self-organizing network and then proposes across-layer network architecture for UAV formation and the requirements of formation and external communication. The development trend of UAV routing protocols is also summarized.
\end{abstract}

Keywords: Unmanned Aerial Vehicle; Network architecture; Routing Protocol

\section{Introduction}

With the rapid development of UAV (unmanned aerial vehicle) technology in the past decade, UAS tend to play an increasingly important role in information acquisition and unmanned operations. It becomes difficult for a single drone to meet the needs of complex tasks, and the application mode of drone formation coordination has gained great attention. First of all, this paper analyzes the structure of self-organizing network and then proposes a cross-layer network architecture for UAV formation and the requirements of formation and external communication. The development trend of UAV routing protocols is also summarized.

In recent years, with the continuous expansion of battle space, countries around the world have launched fierce military competition around a new strategic space. Therefore, UAVs have become an important part of joint operations in space and space integration. A single UAV is subject to many restrictions such as the angle of surveillance, surveillance range, radius of destruction, ability to destroy, and accuracy of attack so that the combat effectiveness is limited. However, the use of UAV formations for coordinated operations can effectively compensate for the lack of operational use of a single UAV. The premise of using UAV formations to conduct coordinated operations is to realize the integration of independent observation, control and communications of the entire formation. The key is that the formation of UAVs must have flexible networking methods and efficient networking capabilities.

Mobile ad hoc network is a multi-hop temporary network composed of mobile nodes. Its non-centered and self-organizing abilities are especially suitable for the collaborative operations of unmanned aerial vehicles in battle field. The maturity of military drone technology and large-scale equipment require the Ad Hoc to possess abilities like building large-scale networks, adapting to high dynamic topology changes, and maintaining good communication performance. The traditional Ad Hoc routing protocol has been difficult to meet the above requirements. Thus, is necessary to develop architecture and routing protocols tailored to the needs of UAV tactical applications. 


\section{Cross-layer network architecture}

Compared with wired networks and traditional wireless networks, mobile ad hoc networks have many different working environments. Therefore, the technologies used are quite different from those of networks. This difference is mainly reflected in the lower three layers of the network: the physical layer, Link layer, network layer. According to the characteristics of mobile ad hoc networks and the OSI reference model. Figure 1 shows the architecture of mobile ad hoc networks.

\begin{tabular}{|c|c|}
\hline \multirow{3}{*}{$\begin{array}{l}\text { High Level Mobile Ad Hoc (WAP, } \\
\text { Internet application, Mobile core } \\
\text { business) }\end{array}$} & Application layer \\
\hline & Presentation layer \\
\hline & Session layer \\
\hline Wireless TCP/UDP & Transport layer \\
\hline Mobile Ad Hoc Network Protocol Layer & Network layer \\
\hline Mobile Ad Hoc Network Data Link Layer & Data link layer \\
\hline Mobile Ad Hoc Network Physical Layer & Physical layer \\
\hline
\end{tabular}

Figure 1 Different layers in Mobile Ad Hoc Network

The network communication protocol often adopts the idea of hierarchical design, that is, the entire communication protocol is divided into several sub-layers which are relatively independent, and all the sub-layers communicate through the defined interfaces. However, the traditional hierarchical network protocol stack is not applicable in the dynamic change of drone tactical networking. Therefore, in order to meet the special requirements of UAV networking, a cross-layer protocol architecture is used to obtain better performance, in which all levels of the protocol stack design have been taken into consideration and arbitrary levels and functional modules are allowed to freely exchange information and feedback between the local changes and other layer to make a reasonable response to adapt to the ad Hoc network dynamic characteristics.

\begin{tabular}{|c|c|c|}
\hline $\begin{array}{c}\text { Transport } \\
\text { layer }\end{array}$ & Speed Control \\
\hline Network layer & $\begin{array}{c}\text { Routine Selection } \\
\text { MAC layer }\end{array}$\begin{tabular}{c} 
Send/Receive Speed \\
Information \\
\hline Physical layer
\end{tabular} Pathway condition \\
\hline
\end{tabular}

Figure 2 Cross-layer structure

The cross-layer structure can effectively meet the application requirements of high dynamic UAV ad hoc networks. Although there are few counterpart studies at present, the next step still has broad research prospects. With the help of the hierarchical structure, the interaction between multiple layers can effectively improve the network performance. For example, the more important physical layer parameters such as the link state information provide an important basis for channel 
intervention and routing forwarding in the upper layer. Another type of thinking is that Multi-layer protocols are merged into a single protocol that can be used for more efficient structural design of UAV ad hoc networks. It is worth noting that it is necessary to pay attention to the universality of cross-layer structures and control of implementation complexity.

\section{Drone ad hoc network Routing Protocol}

Concerning the network architecture, the routing protocol falls into the category of control plane. Through the routing protocol, the network node completes the path selection process, providing the basis and support for end-to-end transmission of user data. Therefore, the performance of the routing protocol will largely affect the quality of outgoing data in the user plane, thereby affecting the service quality of the entire upper-layer application.

So far, dozens of Ad Hoc routing protocols have been proposed, some of which have been adopted by the MANET working group in the IET as a protocol standard. According to different classification criteria, Ad Hoc routing protocols can be divided into different types.

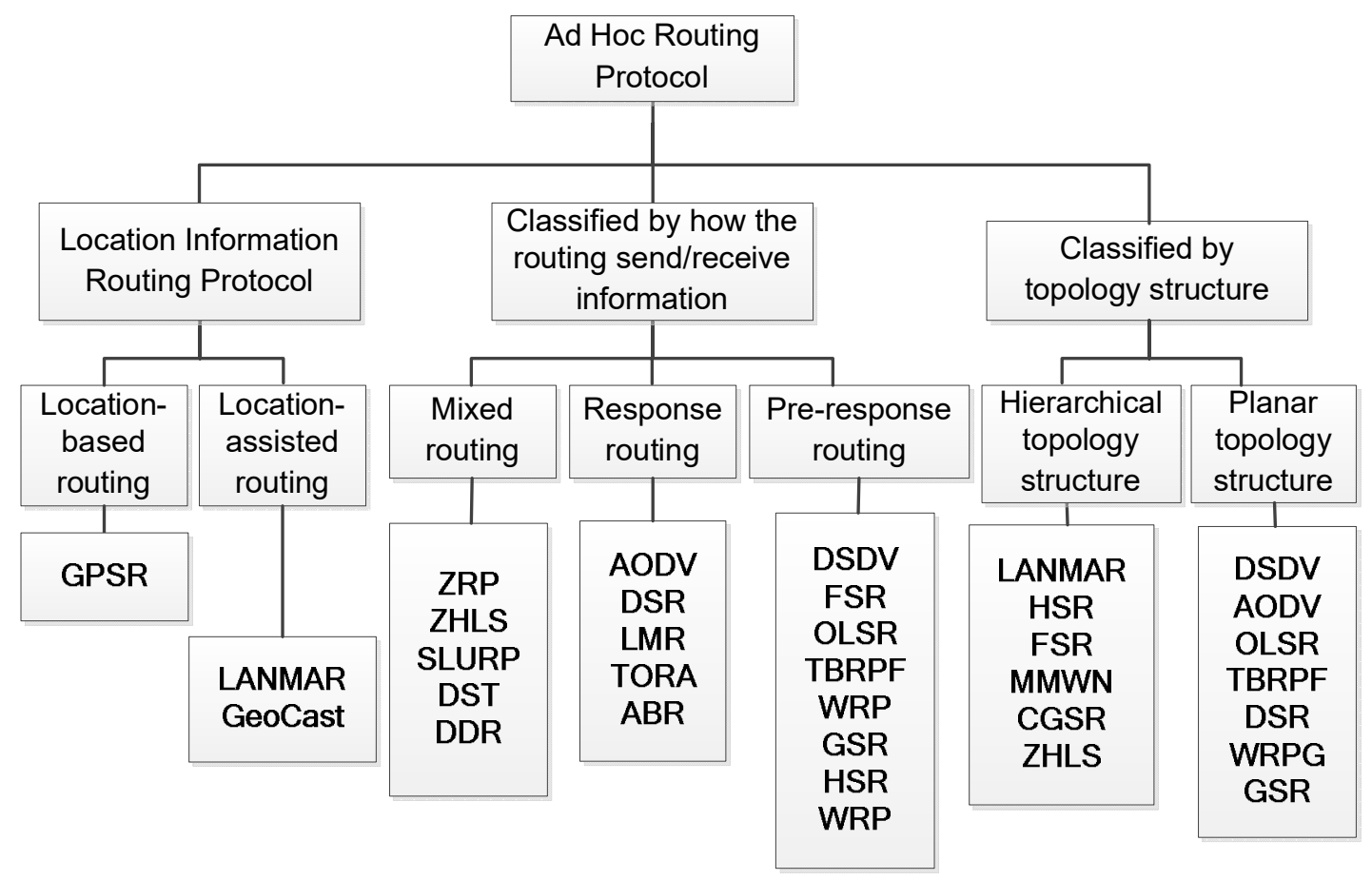

Figure 3 The main types of routing protocols

\section{Future development direction of drone ad hoc network routing protocol}

\section{Geographical location based routing}

With the development of positioning technology, nodes can obtain their own geographical location information. By using these location information, the routing performance of ad hoc networks can be improved. 


\section{Quality of service based routing}

At present, most routing protocols provide best-effort services for business. With the popularity of multi-media applications and the popularity of applications with requirement for certain multi-service quality, QoS routing has become an important issue in the research field of ad hoc networks. However, due to the uncertainty of the ad hoc network topology itself, it is difficult to implement QoS routing in ad hoc networks. In order to achieve QoS maintenance of Ad Hoc networks, several requirements must be satisfied: First, the topology of the network should not change too fast, that is, the frequency of the topology changes should be lower than the frequency at which the topology update information reaches the relevant nodes; secondly, the protocol of the media access layer can support QoS; in addition, it also requires a suitable protocol to support resource management, resource tracking, and status information updates.

\section{Application of Mobile Prediction in High Speed Mobile Networks}

In general communication conditions, the traditional routing protocols generally have the upper limit of its speed by 20 meters per second. Speed higher than 20 will be defined as extreme case. However, in some typical networks, mobile nodes clearly show some kind of mobile mode. Analyzing this non-random motion pattern can predict the network's future topology. In addition, the use of motion prediction can also reduce the number of required two-terminal data packets to reduce overhead. Based on this idea, it is proposed to use GPS as an auxiliary positioning tool. When the links can be connected, the geographical location information is piggybacked to other nodes through the data packets to calculate LET and RET, so that routing is performed before the link breaks according to the information Reconfigure for seamless communication.

\section{References}

[1] Y. B. KO, N. H. Vaidya. Location-aided routing (LAR) in mobile Ad Hoc networks [J]. Wireless Networks, 2000.6(4): 307-321

[2] C. E. Perkins, P. Bhagwat. Highly dynamic destination-sequenced distance-vector routing (DSDV) for mobile computers[J]. Computer Communications Review, 1994, 24(4): 234-244

[3] P. Jacquet, P. Muhlethaler, T. Clausen, et al. Optimized link state routing protocol for ad hoc networks[C]. Multi Topic Conference Proceedings, Lahore, 2001, 62-68

[4] V. D. Park, M. S. Corson. Highly Adaptive Distributed Routing Algorithm for Mobile Wireless Networks[C], 16th IEEE INFOCOM Proceedings, Kobe, 1997, 1405-1414

[5] K. Sanzgiri, B. Dahill, B. N. Levine, et al. A Secure Routing Protocol for Ad hoc Network[C]. 10th IEEE International Conference on Network Protocols Proceedings, Paris, 2002, 78-87

[6] S. Gupte, M. Singhal. Secure routing in mobile wireless ad hoc networks[J]. Ad Hoc Networks, 2003. 1(1): $151-174$

[7] A. S. Alshahrani. Rushing attack in mobile ad hoc networks[C]. Third International Conference on Intelligent Networking and Collaborative Systems, Fukuoka, 2011, 752-758

[8] L. X. Hu, D. Evans. Using directional antennas to Prevent wormhole attacks[C]. Network and Distributed System Security Symposium Proceedings, San Diego, 2004, 1-11 